\title{
LPL methylation of peripheral blood leukocytes - Prophet of atherothrombotic stroke
}

\section{Minjia Xiao ( $018933266 @ q q . c o m$ )}

Second Xiangya Hospital https://orcid.org/0000-0002-5041-2738

\section{Zhijie Xiao}

Second Xiangya Hospital

\section{Research article}

Keywords: lipoprotein lipase methylation, DNA epigenetics, ischemic stroke, atherothrombotic stroke, atherosclerosis, hyperlipidemia, lipid metabolism

Posted Date: March 5th, 2020

DOl: https://doi.org/10.21203/rs.3.rs-16075/v1

License: (1) This work is licensed under a Creative Commons Attribution 4.0 International License. Read Full License 


\section{Abstract}

Background: Lipoprotein lipase $(\mathrm{LPL})$ is a glycoprotein enzyme playing a pivotal role in energy and lipoprotein metabolism. It hydrolyzes triglyceride-rich lipoprotein and produces fatty acids as well as monoacylglycerol. Multiple risk factors including age, sex, hypertension, smoking and hyperlipidemia contribute to ischemic stroke (IS). Hyperlipidemia is an extremely pivotal risk factor for ischemic stroke especially atherothrombotic stroke (ATS) in that it can lead to intracranial or extracranial vessels' atherosclerosis analogous to atherosclerosis of coronary artery. However, potential epigenetic mechanisms contributing to IS are not been explored thoroughly. The aim of this study was to illuminate relationship among individuals' peripheral blood leukocytes methylation level of LPL-promoter-CpG dinucleotides, serum lipid and ATS of Chinese Han population in Hunan Province.

Methods: Peripheral blood samples were collected from acute atherothrombotic stroke patients and healthy people, and methylation level of cytosine-phosphate-guanine $(\mathrm{CpG})$ island in LPL promoter region was measured by qPCR and pyrosequencing. Biochemical and anthropometric variables have also been taken into consideration.

Results: Integral LPL-promoter-CpG dinucleotides methylation level of case group is evidently higher than control group ( $38.8 \pm 8.4 \%$ percentile vs $25.7 \pm 6.6 \%$ percentile, $P$ value $=0.000)$. DNA methylation at the CpG9 16 locus and CpG20 21 locus was related to ATS after adjusting for gender, previous history of diabetes and hypertension, smoking, drinking, body mass index, and blood lipid levels(CpG9 49.3 \pm 24.9 , 31.3 $\pm 13.6, \mathrm{P}$ value $=0.02 ; \mathrm{CpG10} 61.3 \pm 24.5,34.0 \pm 18.4, \mathrm{P}$ value $=0.002 ; \mathrm{CpG} 11$ 71.3 $\pm 17.3,32.0 \pm 21.1, \mathrm{P}$ value $=0.000 ; \mathrm{CpG12} 75.3 \pm 17.7,44.7 \pm 19.6, \mathrm{P}$ value $=0.000 ; \mathrm{CpG13} 72.0 \pm 20.4,50.0 \pm 25.4, \mathrm{P}$ value $=0.014 ;$ CpG14 63.3 $\pm 18.0,45.3 \pm 22.0, P$ value $=0.021 ; C p G 15$ 56.7 $\pm 13.5,34.7 \pm 14.6, P$ value $=0.000 ; C p G 16$ $50.0 \pm 12.5,31.3 \pm 20.0, P$ value $=0.005 ; C p G 20$ 52.0 $\pm 13.2,27.3 \pm 20.9, P$ value $=0.001 ; C p G 2155.3 \pm 11.9$, $34.0 \pm 22.3, P$ value $=0.004)$. There is no statistically significant connection between either carotid atherosclerosis or gender and methylation level. Besides, we found a negative association between LPL methylation status and HDL-C levels, whereas LPL gene methylation was linked with LDL-C levels and age positively.

Conclusion: We illustrate that epigenetic modification of LPL methylation may have an influence on lipids metabolism and occurrence of ATS, which may be a promising indicator for occurrence risk of ischemic stroke. Whether these findings are valid need further warrant and future prospective.

\section{Background}

Epigenetics is a kind of biological phenomenon bringing about heritable alteration while void of DNA sequence change[1]. There are mainly three forms of epigenetic modifications: DNA modification, histone post-translational modifications (PTMs) and chromatin remodeling. Extrinsic factors especially lifestyle and environment can influence the epigenome, for example, exposure to cigarette smoke, nutrients of foods, stress exposure in early life and pollutants exposure in the environment[1].DNA methylation is a 
well-studied and stable epigenetic mark, occurring at position $5^{\prime}$ of the cytosine pyrimidine ring, especially under the circumstance of guanine residues locating next to cytosine residues- CpG dinucleotides, a potential methylation site[2].

Stroke is ranking second among causes of death and adult disability all around the world [3]. Ischemic stroke(IS) accounts for $41-79 \%$ of all strokes (ischemic stroke, intracerebral hemorrhage and subarachnoid hemorrhage) statistically[4]. IS is resulted from arteries' occlusion or stenosis (intracranial or extracranial). It is associated with lifestyle features like smoking, high body-mass index and dietary habits and underlying diseases including hypertension, diabetes and hyperlipidemia. Beyond that, genes and epigenetics are increasingly playing a pivotal role in the pathophysiology[5].

Lipoprotein lipase (LPL) is a rate-limiting enzyme catalyzing triglyceride-rich lipoproteins (TLRs) hydrolysis and decreasing levels of chylomicrons (CM), low-density lipoproteins (LDL) and very lowdensity lipoproteins (VLDL)[6]. It has been proved that LPL plays a role in hypertriglyceridemia and atherosclerotic diseases such as coronary heart disease and atherothrombotic stroke[7], but specific mechanism has not been fully explored yet. We investigate connections of DNA methylation with ATS and correlated atherosclerotic sorts in ATS patients and focus on CpG loci locating in LPL promoter region. To our knowledge, this is the first article to illuminate the relationship between LPL gene methylation and ATS via detecting all of the CpG methylation loci locating in LPL promoter region and display that LPL gene can be an underlying indicator to evaluate occurrence risk of ATS, thus early intervention of gene methylation reversal treatment could be a promising treatment target to reduce ATS risk.

\section{Methods}

In this study, 15 ATS patients hospitalizing in department of neurology and mathed 15 average persons in physical examination center of Second Xiangya Hospital were recruited in 2013. People with coronary heart disease, peripheral vascular disease, aorto-arteritis, hemopathy, tuberculosis, malignant tumor, severe hepatic and renal dysfunction or application of lipid-lowering drugs within 1 week before admission were excluded. Serum samples were fasting venous blood collected at early morning. The study was conducted in accordance with the guidelines laid down in the Declaration of Helsinki. All participants gave their written informed consent, which was obtained in the Ethics approval and consent to participate section. The study was reviewed and approved by the Ethics and Research Committee of Second Xiangya Hospital as well.

\section{Results}

\section{General clinical data comparison of the case group and control group}

Compared with the control group, the case group had higher diastolic blood pressure(DBP) and LDL-C levels while lower HDL-C levels with statistically significant differences $(P<0.05)$. Other general data such 
as gender, age, etc. displayed no significant differences (Table1).

\section{Comparison of LPL methylation level between the case group and control group.}

A total of 30 locus $\mathrm{CpG}$ methylation site were detected in LPL promoter region. In the case group, integral LPL methylation level(38.8 \pm 8.4$)$ was significantly higher in comparison with control group(25.7 \pm 6.6$)$ with statistically differences(P value $<0.05)$, especially at the $\mathrm{CpG9} 16$ locus and CpG20 21 locus(CpG9 49.3 $\pm 24.9,31.3 \pm 13.6, \mathrm{P}$ value $=0.02 ; \mathrm{CpG10} 61.3 \pm 24.5,34.0 \pm 18.4, \mathrm{P}$ value $=0.002 ; \mathrm{CpG} 11$ 71.3 \pm 17.3 , $32.0 \pm 21.1, P$ value $=0.000 ; C p G 1275.3 \pm 17.7,44.7 \pm 19.6, P$ value $=0.000 ; C p G 1372.0 \pm 20.4,50.0 \pm 25.4, P$ value $=0.014 ; \mathrm{CpG14} 63.3 \pm 18.0,45.3 \pm 22.0, \mathrm{P}=0.021 ; \mathrm{CpG} 15$ 56.7 $\pm 13.5,34.7 \pm 14.6$, Pcvalue $=0.000 ; \mathrm{CpG} 16$ $50.0 \pm 12.5,31.3 \pm 20.0, P$ value $=0.005 ; C p G 20$ 52.0 $\pm 13.2,27.3 \pm 20.9, P$ value $=0.001 ; C p G 2155.3 \pm 11.9$, $34.0 \pm 22.3, P$ value $=0.004$ ), while the rest exhibited no significant differences as were manifested in Table2, 3 and Figure1,2.

\section{Comparison of LPL methylation level between individuals with and without cartiod artery atherosclerosis(CAS).}

25 individuals who had undergone cartiod artery ultrasonography were divided into CAS group and nonCAS group. According to the analysis there was no obvious significance of LPL methylation level between the two group $(35.8 \pm 8.7$ vs $30.3 \pm 10.9$, $P$ value $=0.184)$ as was illustrated in Table4.

\section{Relationship among LPL methylation level, serum lipids and age.}

A careful analysis of association between serum lipids and LPL methylation level showed great difference. In order to investigate connection of serum lipids and LPL methylation level, we conducted linear correlation analysis among HDL-C level, LDL-C level and LPL methylation level of all the participants. The results revealed remarkable negative linear correlation between HDL cholesterol level and general LPL methylation level ( $P$ value=0.001).Further analysis of $C p G 1-30$ locus found that linear correlation at CpG9-16 locus was distinctly higher than that of other locus, and the difference was statistically significant $(P$ value $<0.05)$. There was an evident positive linear correlation between $L D L-C$ level and general LPL methylation level $(P$ value $=0.004)$, and the linear correlation between $C p G 10-12$, CpG15 and CpG20 locus were obviously higher than other locus with statistically significant differences ( $P$ value $<0.05$ ).Age is one of the important uncontrollable risk factors for IS. Through analyzing links of age and LPL methylation level, we found a significant positive linear correlation between age and general LPL methylation level ( $P$ value $=0.011)$. Moreover, linear correlation at $C p G$ 9-16 locus were higher than other sites prominently with a statistically significant difference $(P$ value $<0.05)$ as were shown in Table5 and Figure3-5.

\section{Assciation between LPL methylation level and gender}

Individuals in the two groups were further divided into four subgroups on the basis of gender. The results showed no significant difference between male and female either of the case group or the control 
group or the merged group(Table6). However, there existed statistically significant difference(P value $<0.05)$ of LPL methylation level between males/female case group and control group(Table6,7).

\section{Discussion}

Epigenetics have become a crucial character in the pathophysiologic mechanism of cerebrovascular diseases[8]. It is a complex adjustive pathway regulating gene expression without altering genetic code as a response of dynamic environment and exogenous injuries[9]. DNA methylation is the most studied in epigenetics, occurring in $\mathrm{CpG}$ islands by adding a methyl group to cytosine to constitute 5-methylcytosine and subsequent effects are always gene silencing by way of intervening in transcription factors' combination with promoter region[10,11]. The process of DNA methylation is mediated by a group of enzymes called DNA methyltransferases(Dnmts)[11].Previous evidences suggested that DNA hypermethylation was involved in blood-brain barrier and neurovascular unit changes and poor prognosis in stroke[12] and treatment with demethylating agents manifested positive effects on ischemic brain injuries[13,14]. Intriguingly, other clinical study found that DNA hypomethylation was connected with elevated stroke risk and total mortality[15].It was also a better predictor for stroke outcome assessment than chronological age[16]. Alterations of DNA methylation levels of $\mathrm{Na}-\mathrm{K}-\mathrm{Cl}$ cotransporter 1 (NKCC1), thrombospondin1(THBS1), cystathionine-beta-synthase (CBS) and thrombomodulin (TM) had been found in IS pathogenesis[17]. Similarly, DNA methylation in ABCG1 and APOE was correlated with ischemic stroke via disturbed blood lipid levels[18].

LPL plays a pivotal role in the metabolism and transport of lipids as a rate-limiting enzyme. It catalyzes hydrolysis of triglycerides in CM and VLDL thereby affecting serum lipid level[7]. Association between LPL polymorphism and ATS risks in East-Asians was revealed by a meta-analysis[19]. In addition, LPL immune-positive cells were found to assembled around the infarction area and activity of LPL changed dynamically with acute cerebral ischemia-reperfusion in MCAO rat[20]. It had been proved that LPL gene methylation are associated with plasma lipid levels in patients with familial hypercholesterolemia (FH), which might be an underlying molecular mechanism obligated to dyslipidemia[21, 22].Placental LPL methylation levels were associated with gestational diabetes and maternal blood lipids as well[23]. Ana Arpón et al. also detected that LPL methylation in peripheral leukocytes was related to insulin sensitivity in non-diabetic young women[24] D Drogan et al. reported that regional distribution of body fat was in relation to LPL methylation likewise[25]. Moreover, Daniel Castellano-Castillo discovered that adipose tissue LPL methylation was in association with triglyceride concentrations in the metabolic syndrome[26]. But there are no relevant studies focusing on correlations and potential mechanisms between LPL methylation and ATS so far.

As a matter of fact, this is the first article to investigate whether peripheral blood leukocytes' LPL promoter region methylation level is correlative to ATS through exerting influence on serum lipids. In this study, we collected peripheral venous blood as our experiment specimens which is easy to get and preserve and firstly explored relevance of LPL methylation and ATS in virtue of peripheral blood leukocytes. Our results indicated that LPL promoter region hypermethylation was linked with ATS, 
especially at the CpG9 16 locus and CpG20 21 locus. In addition, there existed negative correlation between LPL methylation status and HDL-C levels while positive correlation with LDL-C levels. Otherwise, LPL methylation status was not significantly associated with CAD. In conclusion, we can assume that LPL methylation has effects on patients with ATS by way of bringing about dyslipidemia. LPL methylation level might be a potential predictor forecasting risks of ischemic stroke and modulation of LPL methylation states might reduce stroke risk and ameliorate stroke prognosis as methylated states are reversible. Thus, LPL is a dramatic aim for clinical intervention. There exist some deficiencies in our study as well. The sample size is relatively small and downstream product such as expression level of LPL mRNA and LPL level are deficient. Further investigations are supposed to enlarge sample size and detect upstream and downstream molecules involved in serum lipid metabolism like Dnmts, LPL mRNA and LPL. Besides, follow-up of patients recruited in the case group should be conducted to monitor dynamic change of LPL methylation level as natural course evolve and secondary prevention implement.

\section{Conclusions}

AS is shown above, mean LPL methylation level of case group is higher than control group with statistically significant difference ( $P$ value $=0.000$ ), particularly at $C p G 9 \sim 16$ locus and $C p G 20 \sim 21$ locus (CpG9 P value $=0.02 ; \mathrm{CpG10} \mathrm{P}$ value $=0.002 ; \mathrm{CpG} 11 \mathrm{P}$ value $=0.000 ; \mathrm{CpG12} \mathrm{P}$ value $=0.000 ; \mathrm{CpG} 13 \mathrm{P}$ value $=0.014 ; C p G 14 P=0.021 ; C p G 15 P$ value $=0.000 ; C p G 16 P$ value $=0.005 ; C p G 20 P$ value $=0.001$; CpG21 P value $=0.004)$. No statistically significant is found between either carotid atherosclerosis or gender and methylation level. LPL methylation status has a negative association with HDL-C levels while links positively with LDL-C levels and age. In a word, LPL is a new therapeutic perspective for ischemic stroke especially for atherothrombotic stroke, which needs further study.

\section{Abbreviations}

LPL lipoprotein lipase; IS ischemic stroke; ATS atherothrombotic stroke; CpG cytosine-phosphateguanine; PTMs post-translational modifications; TLRs triglyceride-rich lipoproteins; CM chylomicrons; LDL low-density lipoprotein; VLDL very low-density lipoprotein; DBP blood pressure; CAS cartiod artery atherosclerosis; Dnmts DNA methyltransferases; NKCC1 Na-K-Cl cotransporter 1; THBS1 thrombospondin 1; CBS cystathionine-beta-synthase; TM thrombomodulin; FH hypercholesterolemia

\section{Declarations}

Ethics approval and consent to participate:Research have been performed in accordance with the Declaration of Helsinki and have been approved by ethics committee of Second Xiangya Hospital. All participants have provided their written informed consent for participation, which was obtained in the Ethics approval and consent to participate section.

Consent for publication: Written informed consent for publication of clinical details were obtained from all of the patients included in our study. A copy of the consent form is available for review by the Editor of 
this journal. And we all acknowledged that the Consent for publication statement refers to consent obtained to include any individual person's data in any form (including individual details, images or videos).

Availability of data and material:The datasets analysed during the current study are available from the corresponding author on reasonable request. Competing interests:The authors declare that they have no competing interests. Funding: Not applicable.

Authors' contributions: Xiao MJ:design of the work and analysis and interpretation of data; Xiao ZJ: drafted the work or substantively revised it. All authors have read and approved the manuscript.

Acknowledgements: Not applicable.

\section{References}

1. T, M.A.F.B.B., Chromatin deregulation in disease. Chromosoma, 2016. 125(1): p. 75-93.

2. Y, L.E.Z., DNA Methylation in Mammals. Cold Spring Harbor Perspectives in Biology, 2014. 6(5): p. a019133-a019133.

3. Liu M, W.B., Wang WZ.Lee LM, S.-H. Zhang, and L.-Z. Kong, Stroke in China: epidemiology, prevention, and management strategies. The Lancet Neurology, 2007. 6(5): p. 456-464.

4. Tsai CF, T.B., Sudlow CL, Epidemiology of stroke and its subtypes in Chinese vs white populations: a systematic review. Neurology, 2013. 81(3): p. 264-272.

5. M, F.E.S.U.G.R.P., Systematic Review of Methods and Results of Studies of the Genetic Epidemiology of Ischemic Stroke. Stroke, 2004. 35(1): p. 212-227.

6. Wang, H. and R.H. Eckel, Lipoprotein lipase in the brain and nervous system. Annu Rev Nutr, 2012. 32: p. 147-60.

7. T, H.P.J., et al., Lipoprotein lipase: Biosynthesis, regulatory factors, and its role in atherosclerosis and other diseases. Clinica Chimica Acta, 2018. 480: p. 126-137.

8. Bertogliat, M.J., K.C. Morris-Blanco, and R. Vemuganti, Epigenetic mechanisms of neurodegenerative diseases and acute brain injury. Neurochem Int, 2020. 133: p. 104642.

9. AP, F., Epigenetics at the epicenter of modern medicine. JAMA, 2008. 299(11): p. 1345-1350.

10. Deaton AM, B.A., CpG islands and the regulation of transcription. Genes Dev, 2011. 25(10): p. 10101022.

11. A, J.R.J.T.J., Structure and function of mammalian DNA methy/transferases. Chembiochem, 2011. 12(2): p. 206-22.

12. AV, S.S.P.C.M.-R.G.K.R.A., Involvement of Epigenetic Mechanisms and Non-coding RNAs in BloodBrain Barrier and Neurovascular Unit Injury and Recovery After Stroke. Front Neurosci, 2019. 13: p. 864. 
13. J, C.I.L.C.K.H.C.D.L., Effect of Inhibition of DNA Methylation Combined with Task-Specific Training on Chronic Stroke Recovery. Int J Mol Sci, 2018. 19(7).

14. Matthias Endres, K.R.A.M., et al., DNA Methyltransferase Contributes to Delayed Ischemic Brain Injury. The Journal of Neuroscience, 2000. 20(9): p. 3175-3181.

15. Baccarelli AđWright R囚Bollati, V., et al., Ischemic Heart Disease and Stroke in Relation to Blood DNA Methylation. Epidemiology, 2010. 21(6): p. 819-828.

16. Soriano-Tárraga C, M.-C.M., Giralt-Steinhauer E and R.-C.A. Ois A, Cuadrado-Godia E1, GómezGonzález A1, Vivanco-Hidalgo RM1, Fernández-Cadenas I1, Cullell N1, Roquer J2, Jiménez-Conde J2, Biological age is better than chronological as predictor of 3-month outcome in ischemic stroke. Neurology, 2017. 89(8): p. 830-836.

17. Stanzione, R., et al., Pathogenesis of Ischemic Stroke: Role of Epigenetic Mechanisms. Genes, 2020. 11(1): p. 89.

18. Qin, X., et al., Overall and sex-specific associations between methylation of the ABCG1 and APOE genes and ischemic stroke or other atherosclerosis-related traits in a sibling study of Chinese population. Clinical Epigenetics, 2019. 11(1).

19. Y, W.C.S.T.L.H.B.J.L., Lipoprotein lipase Ser447Ter polymorphism associated with the risk of ischemic stroke: a meta-analysis. Thrombosis research, 2011. 128(5): p. e107-12.

20. Wang X, S.W., Xu E, The expression and activity of brain lipoprotein lipase is increased after acute cerebral ischemia-reperfusion in rats. Neuropathology : official journal of the Japanese Society of Neuropathology, 2010. 30(2): p. 131-9.

21. Guay SP, B.D., Lamarche B, Gaudet D, Bouchard L, Epipolymorphisms within lipoprotein genes contribute independently to plasma lipid levels in familial hypercholesterolemia. Epigenetics, 2014. 9(5): p. 718-729.

22. B, G.S.B.D.L., et al., DNA methylation variations at CETP and LPL gene promoter loci: New molecular biomarkers associated with blood lipid profile variability. Atherosclerosis, 2013. 228(2): p. 413-420.

23. MF, H.A.S.-P.J.H., et al., Placental lipoprotein lipase DNA methylation levels are associated with gestational diabetes mellitus and maternal and cord blood lipid profiles. J Dev Orig Health Dis, 2014. 5(2): p. 132-41.

24. FI, A.A.S.J.M., et al., Insulin Sensitivity Is Associated with Lipoprotein Lipase (LPL) and Catenin Delta 2 (CTNND2) DNA Methylation in Peripheral White Blood Cells in Non-Diabetic Young Women. Int J Mol Sci, 2019. 20(12).

25. Drogan, D., et al., Regional distribution of body fat in relation to DNA methylation within the $L P L$, ADIPOQ and PPARgamma promoters in subcutaneous adipose tissue. Nutr Diabetes, 2015. 5: p. e168.

26. Castellano-Castillo, D., et al., Adipose Tissue LPL Methylation is Associated with Triglyceride Concentrations in the Metabolic Syndrome. Clin Chem, 2018. 64(1): p. 210-218. 


\section{Tables}

Table1. Characteristics of all subjects according to group $(\mathrm{n}=15)$

\begin{tabular}{cccc}
\hline Characteristics & $\begin{array}{c}\text { Case group(n=15) } \\
\text { mean } \pm \text { s.e. }\end{array}$ & $\begin{array}{c}\text { Control group(n=15) } \\
\text { mean } \pm \text { s.e. }\end{array}$ & P value \\
\hline Sex(male/female) & $9 / 6$ & $9 / 6$ & 1.000 \\
Age & $60.7 \pm 10.9$ & $63.3 \pm 11.5$ & 0.530 \\
Height $(\mathrm{cm})$ & $165.7 \pm 8.6$ & $160.7 \pm 6.8$ & 0.090 \\
Weight $(\mathrm{kg})$ & $58.4 \pm 12.2$ & $59.1 \pm 12.3$ & 0.888 \\
BMI $(\mathrm{kg} / \mathrm{m} 2)$ & $21.2 \pm 3.2$ & $22.7 \pm 3.7$ & 0.228 \\
SBP(mmhg) & $152.5 \pm 22.7$ & $137.9 \pm 21.7$ & 0.081 \\
DBP(mmhg) & $94.4 \pm 17.3$ & $77.3 \pm 11.3$ & 0.003 \\
Family history of stroke & $1 / 6.7 \%$ & $1 / 6.7 \%$ & 1.000 \\
Hypertension & $9 / 60.0 \%$ & $5 / 33.3 \%$ & 0.143 \\
Diabetes mellitus & $3 / 20.0 \%$ & $1 / 6.7 \%$ & 0.598 \\
Smoking & $5 / 33.3 \%$ & $2 / 13.3 \%$ & 0.390 \\
Drinking & $2 / 13.3 \%$ & $2 / 13.3 \%$ & 1.000 \\
ALT & $23.4 \pm 10.3$ & $18.3 \pm 5.4$ & 0.102 \\
BUN & $5.7 \pm 1.9$ & $5.4 \pm 1.8$ & 0.744 \\
CREA & $79.5 \pm 27.1$ & $92.4 \pm 19.7$ & 0.146 \\
TG & $2.4 \pm 1.3$ & $1.9 \pm 0.9$ & 0.204 \\
TC & $4.9 \pm 1.2$ & $5.06 \pm 1.2$ & 0.691 \\
HDL-C & $0.9 \pm 0.2$ & $1.2 \pm 0.3$ & 0.017 \\
LDL-C & $3.1 \pm 1.1$ & $2.3 \pm 0.8$ & 0.034 \\
FBS & $7.5 \pm 2.0$ & $6.5 \pm 1.7$ & 0.14 \\
\hline
\end{tabular}

BMI, body mass index; SBP systolic pressure; DBP diastolic blood pressure; ALT, glutamic-pyruvic transaminase; BUN, urea nitrogen; CREA, creatinine; TG, triglyceride; TC, total cholesterol; HDL-C, high-density lipoprotein cholesterol; LDL-C, low-density lipoprotein cholesterol; FBS, fasting blood-glucose. Statistically significant results are shown in bold.

Table2₫ CpG Methylation level at each locus in LPL promoter region 


\begin{tabular}{|c|c|c|c|}
\hline Locus & $\begin{array}{c}\text { Case group }(\mathrm{n}=15) \\
\text { (mean methylation level, CpG \%) }\end{array}$ & $\begin{array}{c}\text { Control group }(\mathrm{n}=15) \\
\text { (mean methylation level, } \mathrm{CpG} \%)\end{array}$ & $\mathrm{P}$ value \\
\hline CpG1 & $28.7 \pm 16.4$ & $24.7 \pm 22.3$ & 0.704 \\
\hline CpG2 & $23.3 \pm 18.8$ & $19.3 \pm 18.7$ & 0.513 \\
\hline CpG3 & $16.0 \pm 15.0$ & $8.7 \pm 13.0$ & 0.141 \\
\hline CpG4 & $32.0 \pm 13.7$ & $23.3 \pm 15.0$ & 0.110 \\
\hline CpG5 & $28.7 \pm 15.5$ & $20.0 \pm 12.0$ & 0.081 \\
\hline CpG6 & $18.7 \pm 11.9$ & $12.7 \pm 11.0$ & 0.178 \\
\hline CpG7 & $34.7 \pm 20.0$ & $26.7 \pm 25.8$ & 0.342 \\
\hline CpG8 & $31.3 \pm 14.6$ & $21.3 \pm 17.3$ & 0.098 \\
\hline CpG9 & $49.3 \pm 24.9$ & $31.3 \pm 13.6$ & 0.020 \\
\hline CpG10 & $61.3 \pm 24.5$ & $34.0 \pm 18.4$ & 0.002 \\
\hline CpG11 & $71.3 \pm 17.3$ & $32.0 \pm 21.1$ & 0.000 \\
\hline CpG12 & $75.3 \pm 17.7$ & $44.7 \pm 19.6$ & 0.000 \\
\hline CpG13 & $72.0 \pm 20.4$ & $50.0 \pm 25.4$ & 0.014 \\
\hline CpG14 & $63.3 \pm 18.0$ & $45.3 \pm 22.0$ & 0.021 \\
\hline CpG15 & $56.7 \pm 13.5$ & $34.7 \pm 14.6$ & 0.000 \\
\hline CpG16 & $50.0 \pm 12.5$ & $31.3 \pm 20.0$ & 0.005 \\
\hline CpG17 & $28.0 \pm 19.0$ & $22.0 \pm 15.7$ & 0.353 \\
\hline CpG18 & $20.0 \pm 13.6$ & $17.3 \pm 15.8$ & 0.624 \\
\hline CpG19 & $30.7 \pm 16.7$ & $24.0 \pm 18.8$ & 0.322 \\
\hline CpG20 & $52.0 \pm 13.2$ & $27.3 \pm 20.9$ & 0.001 \\
\hline CpG21 & $55.3 \pm 11.9$ & $34.0 \pm 22.3$ & 0.004 \\
\hline CpG22 & $28.7 \pm 23.0$ & $16.7 \pm 18.4$ & 0.127 \\
\hline CpG23 & $34.7 \pm 23.9$ & $24.7 \pm 24.8$ & 0.322 \\
\hline CpG24 & $24.7 \pm 15.5$ & $16.7 \pm 15.0$ & 0.180 \\
\hline CpG25 & $36.0 \pm 17.7$ & $26.0 \pm 18.8$ & 0.145 \\
\hline CpG26 & $48.7 \pm 15.1$ & $40.7 \pm 23.1$ & 0.271 \\
\hline CpG27 & $30.0 \pm 16.0$ & $20.0 \pm 18.1$ & 0.171 \\
\hline CpG28 & $35.3 \pm 14.6$ & $24.0 \pm 16.8$ & 0.059 \\
\hline CpG29 & $12.7 \pm 10.3$ & $8.0 \pm 14.2$ & 0.103 \\
\hline CpG30 & $14.0 \pm 11.2$ & $8.7 \pm 14.1$ & 0.128 \\
\hline
\end{tabular}

Statistically significant results are shown in bold.

Table3₫ Mean CpG methylation level in case group and control group

\begin{tabular}{cccc}
\hline & Case group & Control group & P value \\
\hline Sum & 15 & 15 & \\
Mean methylation level, CpGZ \% & $38.8 \pm 8.4$ & $25.7 \pm 6.6$ & $0.000^{* *}$ \\
\hline
\end{tabular}

Statistically significant results are shown in bold, ${ }^{* *} P \llbracket 0.01 \square$

Table4. LPL methylation level of CAS group and non-CAS group

\begin{tabular}{cccc}
\hline & CAS group & non-CAS group & P value \\
\hline Sum & 9 & 16 & \\
Mean methylation level (CpGZ \%) & $35.8 \pm 8.7$ & $30.3 \pm 10.9$ & 0.184 \\
\hline
\end{tabular}


本

Table5. Relationship among LPL methylation level, serum lipids and age

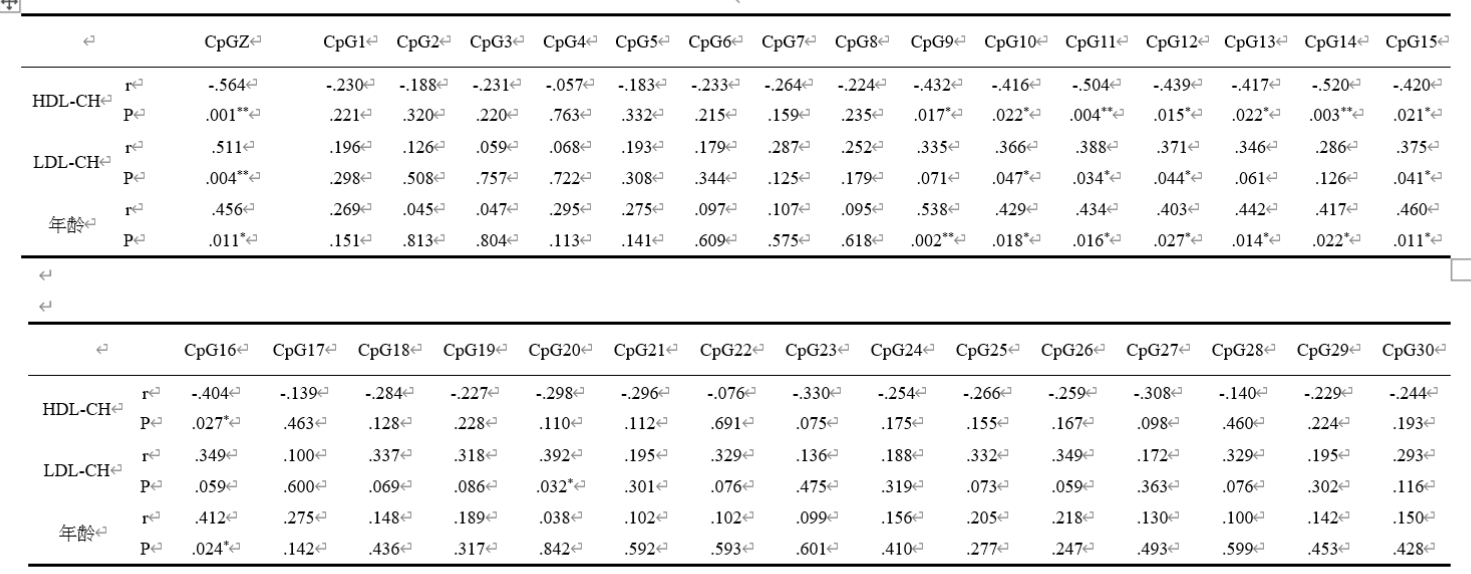

*. Correlation is significant at the 0.05 level

**. Correlation is significant at the 0.01 level

Table6. Assciation between LPL methylation level and gender

6

\begin{tabular}{cccccccc}
\hline & \multicolumn{2}{c}{ Male } & & \multicolumn{2}{c}{ Female } & P value \\
\cline { 2 - 3 } & Sum & CpGZ\% & & Sum & CpGZ\% & \\
\hline Case & 9 & $37.3 \pm 7.9$ & & 6 & $41.0 \pm 9.5$ & 0.426 \\
Control & 9 & $24.0 \pm 6.1$ & & 6 & $28.2 \pm 7.1$ & 0.246 \\
& & & & & & \\
Merged & 18 & $30.7 \pm 9.7$ & & 12 & $34.6 \pm 10.4$ & 0.299 \\
\hline
\end{tabular}

Table7. Assciation between LPL methylation level and gender

\begin{tabular}{cccccccc}
\hline & \multicolumn{2}{c}{ Case group } & & \multicolumn{3}{c}{ Control group } & P value \\
\cline { 2 - 3 } \cline { 5 - 6 } & Sum & CpGZ\% & & Sum & CpGZ\% & \\
\hline Male & 9 & $37.3 \pm 7.9$ & & 9 & $24.0 \pm 6.1$ & $0.001^{* *}$ \\
Female & 6 & $41.0 \pm 9.5$ & & 6 & $28.2 \pm 7.1$ & $0.024 *$ \\
& & & & & & \\
\hline
\end{tabular}

Statistically significant results are shown in bold, ${ }^{*} P[0.05 \square * * P \square 0.01$

\section{Figures}




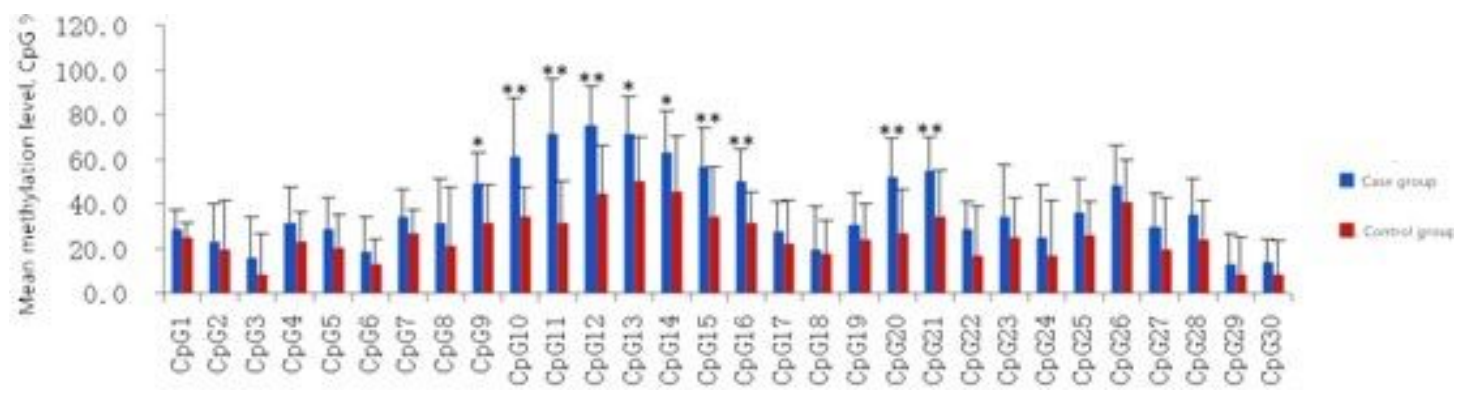

Figure 1

CpG Methylation level at each locus in LPL promoter region

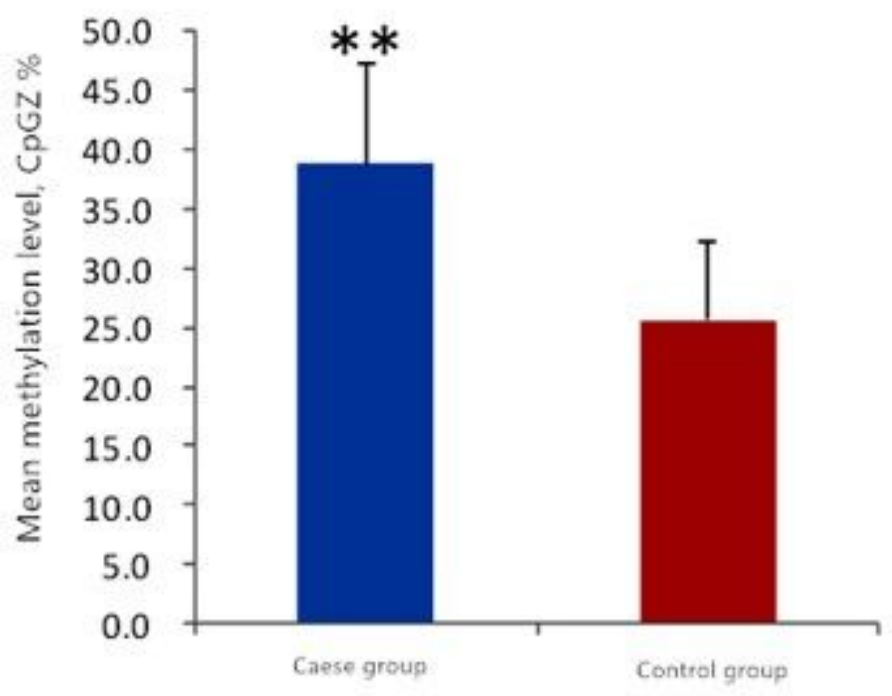

${ }^{* *} \mathrm{P}$ value $<0.01$

Figure 2

Mean CpG methylation level in case group and control group 


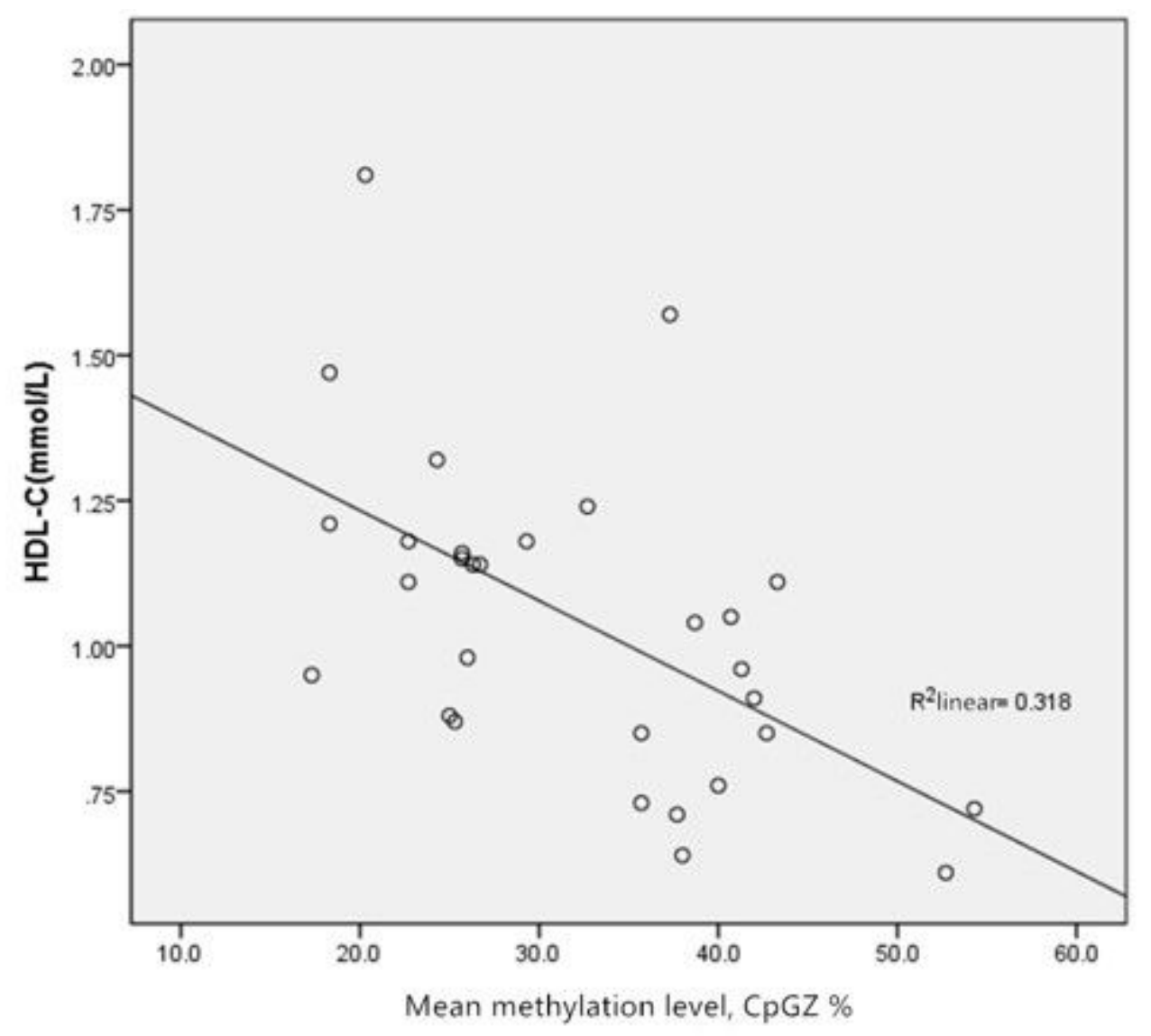

Figure 3

Relationship between LPL promoter mean methylation level and HDL-C

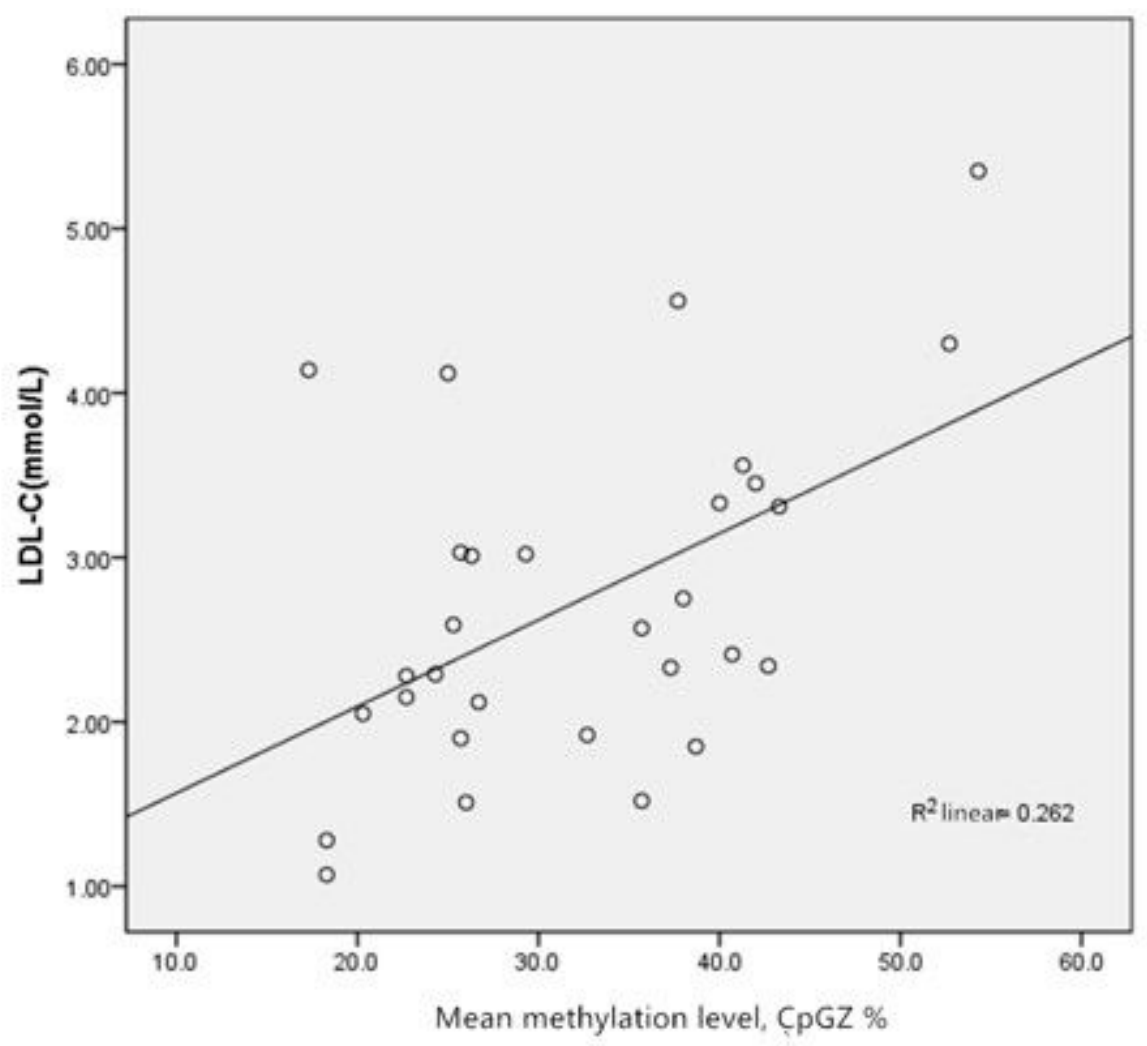


Figure 4

Relationship between LPL promoter mean methylation level and LDL-C

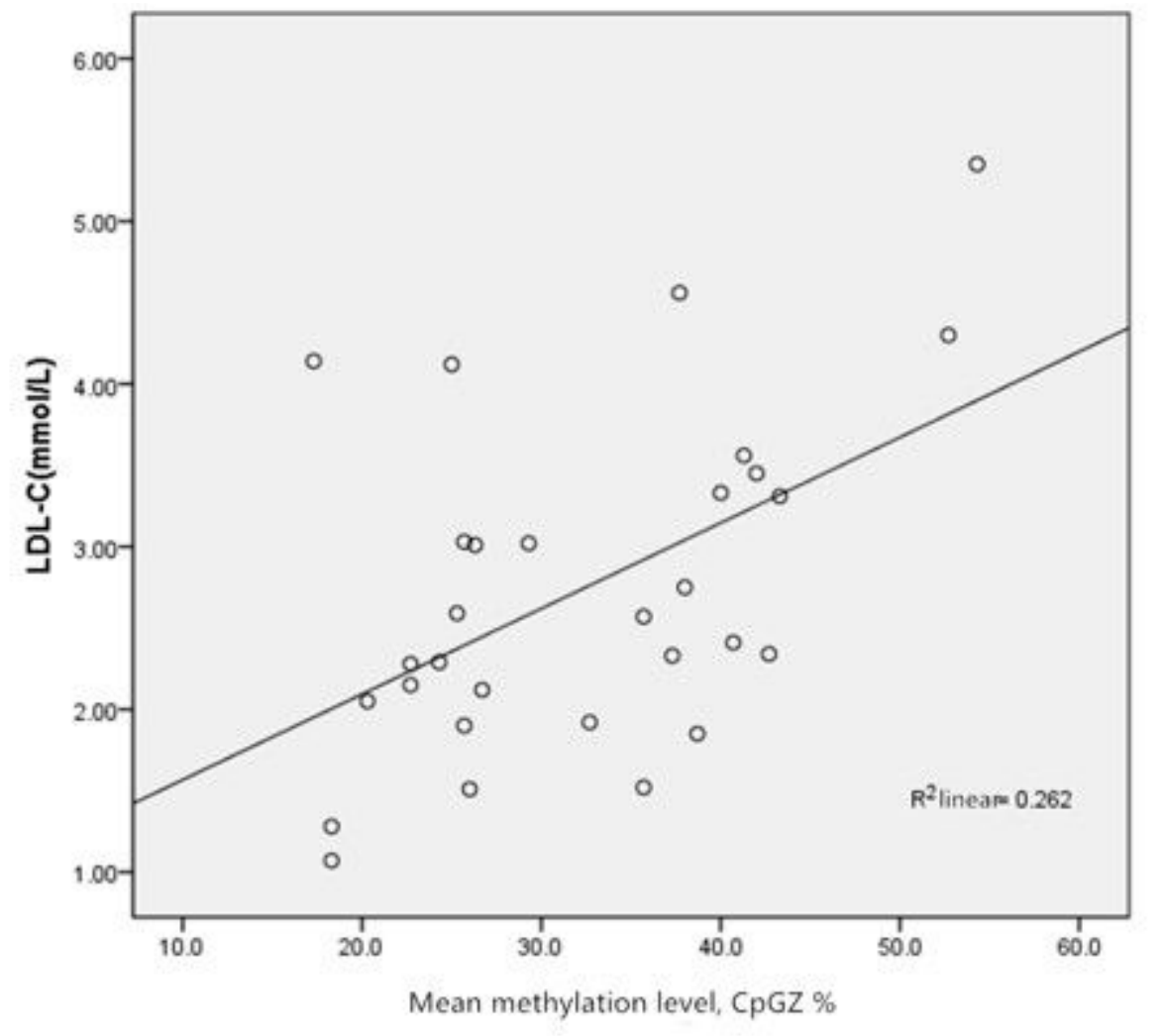

Figure 5

Relationship between LPL promoter mean methylation level and age 